\title{
Anti-phase synchronization of electrically shaken conducting beads
}

\author{
E. Mersch and N. Vandewalle \\ GRASP, Institut de Physique B5, \\ Université de Liège, B-4000 Liège, Belgium.
}

\begin{abstract}
When a spherical conducting bead is placed in an electrode, it experiences an electric force. In a plane capacitor, it can undergo a periodic bouncing between the electrodes. Using a fast video camera, we measured the acceleration of the bead and the period of its motion as a function of the applied voltage. A mathematical model based on the hypothesis of electrostatic equilibrium is proposed to describe the dynamics of the system. We observe a stabilization of the trajectories : a bead bouncing between two electrodes tends to oscillate on a quasi-vertical trajectory, whatever its initial horizontal velocity. When two identical beads are placed together in a capacitor, they oscillate at the same frequency and an anti-phase synchronization effect occurs. We propose a simple mechanism based on a Kuramoto-like model to explain it.
\end{abstract}

PACS numbers: 45.70.-n, 45.50.-j, 05.45.Xt, 41.20.Cv

\section{Introduction}

When micron or millimeter-sized objects are placed in contact with an electrode, they can experience forces larger than their weight. The dynamics of a conducting bead immersed in a poorly conducting liquid inside a horizontal plane capacitor submitted to a DC voltage was studied by Khayari and coworkers [1]. When charged on the bottom plate, the ball is pushed in the upward direction. After its discharge through the liquid, the it goes back down to the bottom electrode because of gravity. The system exhibits a periodic dynamics. The same experiment was conducted with an additional alternating electric field [2]. When increasing the amplitude of the alternating electric field, the system exhibits a period-doubling bifurcation analogous to the one observed for a ball mechanically shaken on an horizontal plate.

For a larger number of particles, the mutual interactions are responsible for some fascinating collective behaviors. Saint Jean and coworkers studied the diffusion of millimeter-sized spherical conducting particles placed between electrodes of different geometries and submitted to a mechanical shaking. They used this system to study the structure and the melting of small Wigner crystals in 2D [3-7]. They also studied the single-file diffusion problem within this system [8-10]. Aranson and coworkers[11-19] studied some collective behaviors of large assemblies of conducting beads and glass beads of various diameters enclosed in plane capacitors. They studied the formation dynamics of clusters and could apply the attachment-detachment-controlled Ostwald ripening theory to describe it [11-15]. They studied the velocity distributions of this far from equilibrium system [16] as well as some pattern formation when micron-sized conducting spheres are immersed in a poorly conducting liquids in the presence of a dc electric field [17-19]. Zhand and Liu studied the effect of an alternating electric field on a very similar experiment [20].

In this work, we focused our attention on a minimalist version of the experiment of Aranson and co-workers. We studied the behavior of a single, two or three spherical conducting beads submitted to a constant and homogeneous electric field. In section I, we describe our experimental setup. The section
II is dedicated to the problem of a single bead. In section III, we discuss the dynamics of two interacting beads.

\section{EXPERIMENTAL SETUP}

A sketch of our experimental setup is shown in Figure 1. One, two or three spherical beads are placed between two horizontal plane electrodes. For the experiments conducted with a single bead, we used two glass beads with respective diameters $d=400 \mu \mathrm{m}$ and $d=488 \mu \mathrm{m}$ coated with a conducting layer as well as a steel bead of diameter $d=500 \mu \mathrm{m}$. For the experiment with two or three beads we used two stainless steal beads with a diameter $d=2 \mathrm{~mm}$. The plates of the capacitor are two parallel square with an edge $D=60 \mathrm{~mm}$ separated by a fixed gap $h=3 \mathrm{~mm}$. A DC voltage $V$ comprised between 0 and $5000 \mathrm{~V}$ is applied to the electrodes. The cell is open in an atmosphere of controlled humidity $(43 \% \mathrm{RH})$ at ambient pressure and temperature. The choice of this humidity is motivated by a recent work [21] demonstrating a minimum of bead-bead cohesion induced by capillary condensation when $R H \approx 40-50 \%$. A fast CCD camera records the trajectory from the side at a frame rate ranging between 500 and $6000 \mathrm{fps}$.

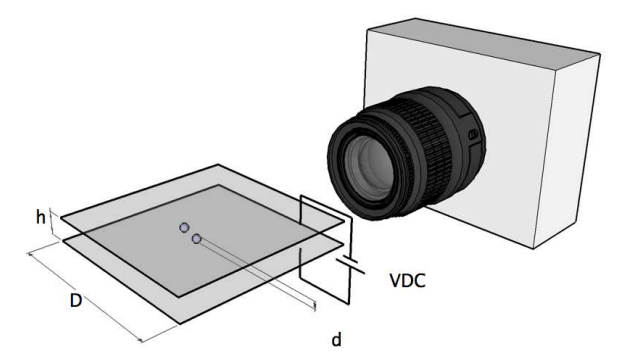

FIG. 1: Sketch of the experimental setup. One or two spherical conducting beads are placed between two horizontal plane electrodes plugged to a high voltage power supply. A fast CCD camera and a tracking algorithm are used to record the trajectory of the beads. 


\section{DYNAMICS OF A SINGLE BEAD}

\section{A. Experimental results}

At the beginning of the experiment, the bead is placed in contact with the bottom electrode. If the applied electric field is large enough, the particle can detach and accelerate in the direction of the upper electrode. It collides with this electrode. During the bouncing, the bead charges with the opposite sign and it further accelerates in the opposite direction. A periodic motion with a limit cycle is thus reached. The Figure 2 represents the vertical coordinate $z$ of a $488 \mu \mathrm{m}$ coated glass bead as a function of time $t$. The applied voltage $V$ is $2000 \mathrm{~V}$ and the frame rate is $6000 \mathrm{fps}$. The limit cycle is reached after about 10 collisions, which correspond to $50 \mathrm{~ms}$.

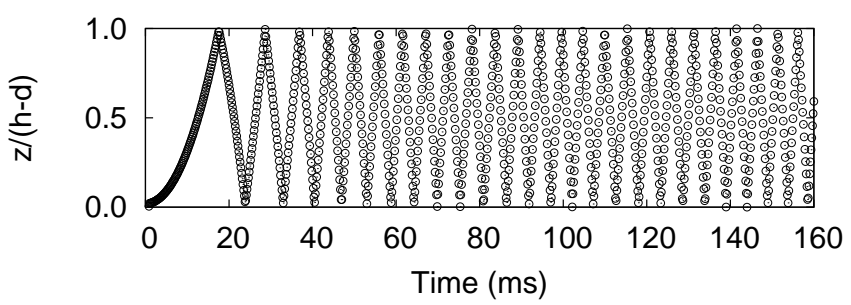

FIG. 2: Temporal evolution of the vertical coordinate $z$ of a single coated glass bead of $488 \mu \mathrm{m}$ under $2000 \mathrm{~V}$. $z$ is normalized by $h-d$ in order to obtain values in the interval $[0,1]$.

We recorded the trajectories of a single $400 \mu \mathrm{m}$ coated glass bead. We measured its acceleration for different voltage values. We obtained the acceleration $a$ due to the electric force by subtracting the gravitational acceleration $g=9.81 \mathrm{~m} \mathrm{~s}^{-2}$. The Figure 3 shows the acceleration $a$ as a function of the applied voltage $V$. Each dot is an average of 5 samples. The acceleration increases with the voltage $V$ in a quadratic way (see model in section II B). The acceleration $a$ reaches values up to $25 \mathrm{~g}$. Three regions denoted I, II and III correspond to three dynamical regimes described in section II B.

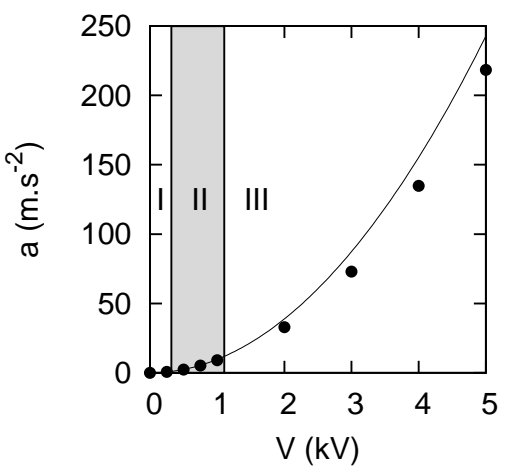

FIG. 3: Measured acceleration $a$ of a coated glass bead of $400 \mu \mathrm{m}$. The solid curve represents the Eq.(4), without any fitting parameter. Three regions I, II, III represent three dynamical regimes described in section II B.

We measured the period $T$ of the limit cycle of a $488 \mu \mathrm{m}$ coated glass bead. The Figure 4 presents the period $T$ as a function of the applied voltage $V$. Each dot is an average computed over 20 to 50 oscillations. As expected form the above observations, the period decreases with increasing voltage.

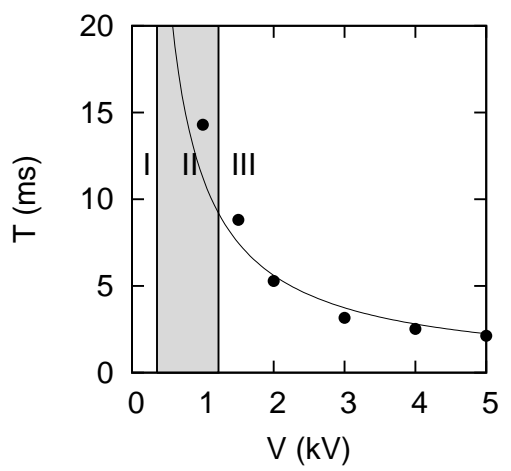

FIG. 4: Measured period $T$ of the limit cycle of a coated glass bead of $488 \mu \mathrm{m}$. The solid curve represents the Eq.(6) without any fitting parameter. Three regions I, II, III represent three dynamical regimes described in section II B.

When a horizontal velocity is given to a bead bouncing on the bottom plate, it keeps a finite horizontal velocity for a long time. On the other hand, when a bead bounces alternatively on both plates, it rapidly reaches a quasi-vertical oscillation. A horizontal stabilization effect occurs for a bead bouncing alternatively on two facing plates (see Figure 5). The mechanism for this stabilization is not trivial. However, we can understand that when a bead is bouncing between two plates, the couple of the friction force can change of sign at each bounce and tend to reduce the rotation of the bead. This is not the case for a bead bouncing only on the bottom plate.

By drawing a mark on the beads, we could observe their rotation. When a bead is bouncing between two plates with a non zero horizontal component of velocity, its angular rotation speed is initialy of the order of $15 \mathrm{rad} / \mathrm{s}$. During the collisions, the sign of angular rotation can change. After a few collisions, the angular velocity vanishes rapidly. However, when the bead is bouncing on the bottom plate only, the sign of the angular rotation remains unchanged. The angular velocity slowly vanishes.

This effect was already reported by Leconte and coworkers [26] for a bead mechanically shaken between two parallel plates. They took advantage of this to make precise measurements of the normal restitution coefficient of a steal bead. It also plays a crucial role for the experiment described in the section III of this article.

\section{B. Mathematical description}

In the following, we will suppose that the charging time of the conducting bead is shorter than the time of contact with an electrode. This means that the electrostatic equilibrium is reached during each contact. The damping due to viscous friction with air is negligible. 

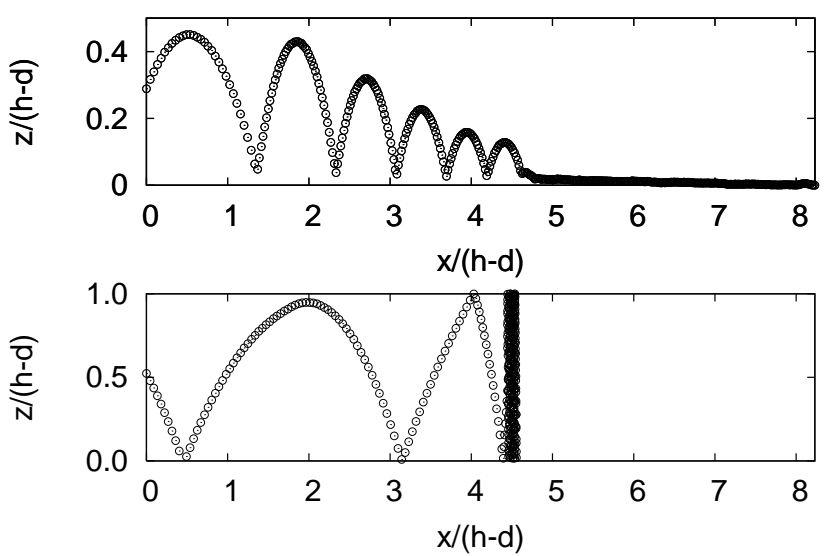

FIG. 5: Horizontal stabilization of a bouncing bead. The horizontal velocity of a bead bouncing on the bottom plate is slowly reduced (top picture). The horizontal velocity of a bead bouncing between two plates is rapidly reduced and the trajectory becomes quasivertical (bottom picture). Both pictures correspond to the same bead, to the same applied voltage, and the same video length.

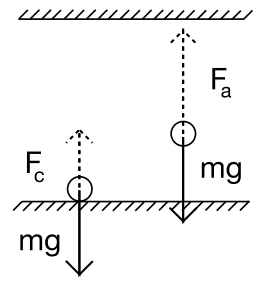

FIG. 6: When a bead is in contact with an electrode, it experiences its weight and a force $F_{c}$ given by Eq.(1). When it is far from the electrodes, it experiences its weight and an electric force $F_{a}$ given by Eq.(3).

First let us calculate the force $F_{c}$ that acts on a bead when it is in contact with an electrode. If the diameter $d$ of the bead is small compared to the gap $h$ between the electrodes, we can refer to the problem of a sphere in contact with a plane placed at the same electric potential. According to Maxwell [22], $F_{c}$ reads

$$
F_{c}=\pi c \epsilon_{0} d^{2} E_{0}^{2}
$$

where $\epsilon_{0}$ is the vacuum permittivity, $E_{0}=V / h$ is the applied electric field, $\rho$ the density of the bead and $d$ its diameter, $c \approx$ 1.36 is an integration constant. If this force is larger than the weight of the bead, it can not stay on the bottom plate and it reaches the limit cycle. The critical electric field $E_{2}$ for this force balance is given by

$$
E_{2}=\sqrt{\frac{\rho g d}{6 c \epsilon_{0}}} .
$$

This expression was used by Aranson and co-workers, and it appears as a critical value on the phase diagrams of several experiments conducted with a large number of particles [1114]. When the bead accelerates between the electrodes, it experiences an electric force $F_{a}$. This force is not anymore given by Eq.(1). If the diameter $d$ is small compared to the gap $h$, we can consider that during the trajectory between the electrodes, the bead is submitted to a force of the form :

$$
F_{a}=q E_{0},
$$

where $q$ is the total charge on the bead. The exact expression of this charge has been calculated in [22]. We use it to express $F_{a}$ as

$$
F_{a}=\frac{\pi^{3} \epsilon_{0} d^{2} E_{0}^{2}}{6}=\frac{\pi^{2}}{6 c} F_{c} .
$$

A more accurate expression of the force acting on a conducting sphere close to a plane electrode can be found in [23]. The forces $F_{a}$ and $F_{c}$ are sketched in Figure 6 . When the bead is going upwards, it is submitted to an acceleration $a-g$, where $g$ is the gravitational acceleration. When it goes downwards, it is submitted to an acceleration $-a-g$. The acceleration $a$ due to the force $F_{a}$ reads

$$
a=\frac{\pi^{2} \epsilon_{0} E_{0}^{2}}{\rho d}
$$

After some collisions, the bead reaches a periodic limit cycle. During one period of this limit cycle, the energy given by the work of the electric force is counterbalanced by the energy dissipated during the collisions. The period $T$ of the limit cycle can therefore be expressed as

$$
\begin{aligned}
T & =\left(\frac{\sqrt{2}}{a+g}-\frac{e \sqrt{2}}{a-g}\right) \sqrt{\frac{(h-d)\left(a+g+a e^{2}-g e^{2}\right)}{1-e^{4}}} \\
& +\left(\frac{\sqrt{2}}{a-g}-\frac{e \sqrt{2}}{a+g}\right) \sqrt{\frac{(h-d)\left(a-g+a e^{2}+g e^{2}\right)}{1-e^{4}}} .
\end{aligned}
$$

If $a \gg g, T$ can be expressed as

$$
T \approx \frac{\sqrt{8}}{\pi} \sqrt{\frac{1-e}{1+e} \frac{\rho d(h-d)}{\epsilon_{0} E_{0}^{2}}} .
$$

The limit cycle can still be stable if $F_{c}<m g$. There is another critical field $E_{1}$ below which the limit cycle becomes unstable. This critical electric field writes

$$
E_{1}=\sqrt{\frac{1-e^{2}}{1+e^{2}} \frac{\rho g d}{\pi^{2} \epsilon_{0}}}=\sqrt{\frac{1-e^{2}}{1+e^{2}} \frac{6 c}{\pi^{2}}} E_{2} .
$$

The dynamics of the system is therefore determined by three regimes : 
- In the region I $\left(E_{0}<E_{1}\right)$ : the only stable attractor is a fixed point : the bead stays on the bottom plate.

- In the region II $\left(E_{1}<E_{0}<E_{2}\right)$ : they are two stable attractors : a periodic limit cycle and a fixed point.

- In the region III $\left(E_{0}>E_{2}\right)$ : the fixed point loses its stability and the limit cycle is the only stable attractor.

The predictions of this model are respectively plotted in Figures 3 and 4. The acceleration $a$ (Eq.(4)), the period $T$ (Eq.(6)) and the critical electric fields $E_{1}$ and $E_{2}$ (Eqs.(7) and (2)) are predicted without fitting parameter. We used $\rho=2500 \mathrm{~kg} \mathrm{~m}^{-3}, d=400 \mu \mathrm{m}$ and $d=488 \mu \mathrm{m}$. The normal restitution coefficient $e=0.905$ was measured independently. The values $E_{1}$ and $E_{2}$ were measured for a steel bead of diameter $d=500 \mu \mathrm{m}$. The value $E_{2}$ is correctly predicted by Eq. 2 . The experimental value of $E_{1}$ is however larger than the one predicted by Eq. 7. When decreasing the electric field above $E_{1}$, small fluctuations of the bead velocity may cause the loss of stability of the oscillations. Experiments and theory are in agreement. The small deviations of the master curve in Figure 4 is probably due to an effect of projection due to the position of the camera.

The dynamics of a single bead electrically shaken is more simple than the dynamics of a bead mechanically shaken between two oscillating plates. Only two attractors are observed for electrical shaking, whereas bifurcation cascade and chaotic orbits are expected for mechanical shaking [24, 25].

\section{DYNAMICS OF SEVERAL INTERACTING BEADS}

\section{A. Two beads}

In this section, we describe the experiment of two neighboring identical beads in a capacitor. The beads are stainless steel beads with a diameter $d=2 \mathrm{~mm}$. The applied voltage $V$ is $2000 \mathrm{~V}$. The frame rate of the video camera is $2000 \mathrm{fps}$.

Figure 7 shows a typical evolution of the height $z$ of two neighboring beads. The beads oscillate with the same frequency, and after few collisions with the plates, they get in phase opposition. The horizontal stabilization effect described in section II A is crucial for this experiment : both beads need to keep a constant frequency and to stay close to each other to become anti-synchronized.

In order the measure a phase shift between the experimental trajectories of the beads, we defined the following parameter :

$$
\phi(t)=\cos ^{-1}\left(\frac{\left\langle\left(z_{1}(t)-\bar{z}_{1}\right)\left(z_{2}(t)-\bar{z}_{2}\right)\right\rangle_{T}}{\sqrt{\left\langle\left(z_{1}(t)-\bar{z}_{1}\right)^{2}\right\rangle_{T}\left\langle\left(z_{2}(t)-\bar{z}_{2}\right)^{2}\right\rangle_{T}}}\right)
$$

where $z_{i}$ designates the vertical coordinate of the bead $i$, $i \in\{1,2\}, \bar{z}_{i}$ the average of $z_{i}$ over all the total length of

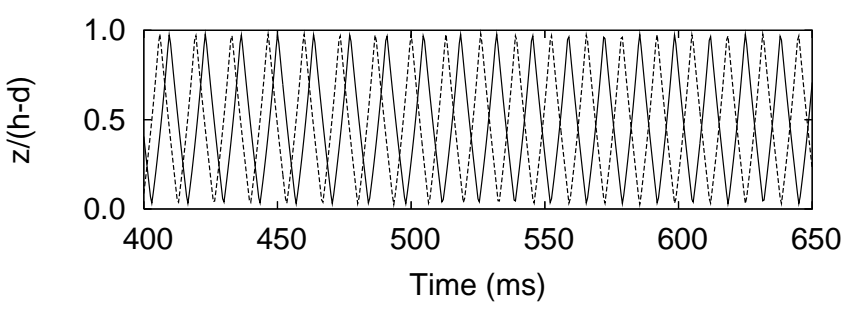

FIG. 7: Temporal evolution of the vertical coordinate of two interacting steal beads of $2 \mathrm{~mm}$ diameter under a voltage of $2000 \mathrm{~V}$. The beads oscillate at the same frequency and get in phase opposition.

the video, and the bracket $\langle f(t)\rangle_{T}$ denotes the mobile average $\int_{0}^{T} f(t+\tau) d \tau$ of the function $f$ over one period $T$.

Figure 8 illustrates the temporal evolution of the phase shift $\phi$ and the horizontal separation $x_{1}-x_{2}$ between the beads. The total time of the sample shown in this picture $(1050 \mathrm{~ms})$ corresponds to 75 oscillations. After some time, $\phi$ reaches a horizontal plateau, indicating a phase locking at a phase shift of $\pi$. When the beads are synchronized, they are attracted by each other and they undergo a collision. After the collision, the separation between the beads is rapidly stabilized and they undergo a second collision. This is the sign of the stabilization effect described in section II A. This behavior is typical for a system of two identical beads. We repeated the measurements of $\phi$ several times, the horizontal plateau of $\phi$ is reproducible, and it always appears for a phase shift of $\pi$.

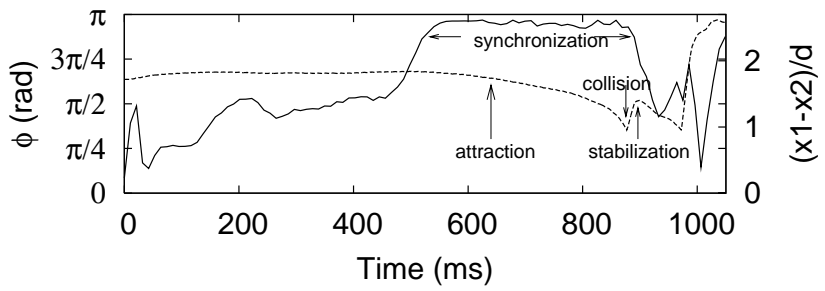

FIG. 8: Typical temporal evolution of the phase shift $\phi$ (solid line) and horizontal separation $\left(x_{1}-x_{2}\right) / d$ (dashed line) for two neighboring beads. One observes a phase synchronization for $\phi=\pi$, during which the beads are attracted by each other. After some time, the beads collide and are stabilized horizontally. The corresponding vertical coordinates $z$ are illustrated by the Figure 7 for the time interval $t \in[400,650]$.

\section{B. Mathematical description}

We can propose a simple mechanism for the anti-phase synchronization described in the previous subsection. Let us consider two beads 1 and 2 and let us define two phases $\theta_{1}$ and $\theta_{2}$ by $\cos \theta_{i}=1-\frac{2 z_{i}}{h-d}, i \in\{1,2\}$. Let us suppose that, without interaction, we have $\dot{\theta_{1}}=\dot{\theta_{2}}=\omega$. When the beads are close to each other, they interact electrostatically. We know that the charge acquired by a bead in contact with an electrode is proportional to the electric field. We also know that 
after the detachment, the acceleration of the bead is proportional to its charge. When two beads are close to each other, the local electric field is modified by the presence of the other bead. The charge acquired by a bead during a contact therefore depends on the phase of the other bead. This mechanism is illustrated by Figure 9 .

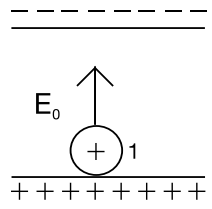

$\dot{\theta}_{1}=\omega$

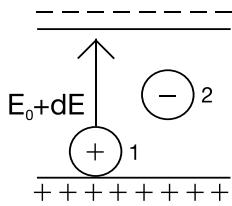

$\dot{\theta}_{1}=\omega+\mathrm{d} \omega$

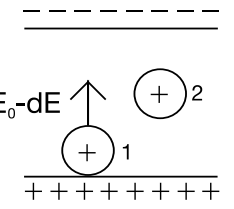

$\dot{\theta}_{1}=\omega-\mathrm{d} \omega$
FIG. 9: The local electric field during the charging of a bead is modified by the presence of the other bead. The acquired charge and the further acceleration of this bead depends on the phase of the other bead.

By considering the sign of the variation of the local electric field for several values of $\theta_{1}$ and $\theta_{2}$, we can write an expression that qualitatively describes the evolution of the phase of the interacting beads. This gives the following Kuramoto-like equations :

$$
\left\{\begin{array}{l}
\dot{\theta_{1}}=\omega+K\left(\beta-\cos \theta_{1} \sin \theta_{2}\right) \\
\dot{\theta_{2}}=\omega+K\left(\beta-\cos \theta_{2} \sin \theta_{1}\right),
\end{array}\right.
$$

where $K$ and $\beta$ are two positive coupling constants. From this expression, we can deduce an equation of evolution for the phase shift $\phi=\theta_{1}-\theta_{2}$.

$$
\dot{\phi}=K \sin (\phi)
$$

The only stable stationary solution of this equation implies

$$
\phi=(2 k+1) \pi,
$$

with $k$ being an integer number. This minimalist Kuramotolike model predicts some synchronization effect. Only an antiphase situation is predicted, as observed experimentally.

\section{Three beads}

We performed experiments on a set of three beads aligned or arranged in a triangle. Only a partial synchronization was observed : two beads could synchronize with a phase shift of $\pi$, similarly to subsection III A, while the phase shift between the other couples of beads fluctuates (see Figure 10).
Experiments with more than two beads are not obvious since the interdistance should be finely controlled in order to induce interactions between the bodies. When more than two beads are present, synchronization appears only for couples of neighboring objects. Since beads are freely moving, reorganization destroys any ordered pattern. This effect could play a major role in the collective motion of beads in between two plates. But the investigation of these collective effects for many beads is outside the scope of the present paper.

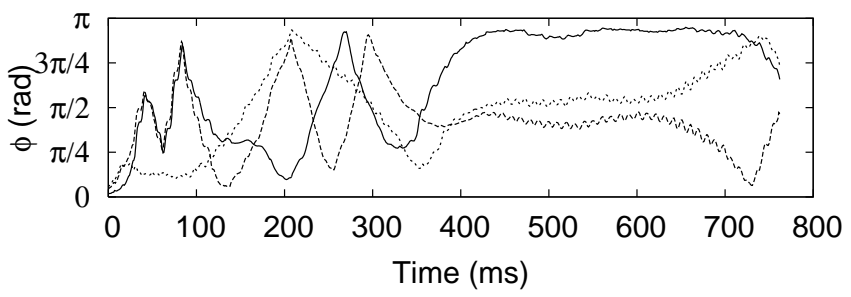

FIG. 10: Temporal evolution of the phase shifts $\phi$ between the pairs of beads in a configuration of three aligned beads. One observes a partial synchronization for $\phi=\pi$ for a pair of beads (plain curve) while the other (dashed curves) are not synchronized.

\section{CONCLUSION}

We performed some experiments concerning the dynamics of spherical conducting particles in a plane capacitor. For a single bead, there are two stable attractors : the bead can stay on the bottom electrode or it can bounce periodically between both electrodes. The acceleration of the bead and the period of its limit cycle were measured as a function of the applied voltage. We propose a zero-fitting parameter model which is in agreement with the experiments. This model also predicts the stability limits for both attractors. Moreover, a horizontal stabilization effect is observed : a bead bouncing between two electrodes tends to reach a quasi-vertical trajectory, whatever its initial horizontal velocity. Thanks to this effect, when two identical beads are placed together in a capacitor, an antiphase synchronization can be observed. We suggest that this synchronization is due to the modification of the local electric field during the charging of the beads. A Kuramoto-like model is proposed, which represents a basis for further studies.

\section{Acknowledgements}

This work has been supported by INANOMAT project (Grant No. IAP P6/17) of the Belgian Science Policy. We acknowledge J-C. Remy, F. Bochini, R. Cloots, G. Lumay and F. Ludewig for help and fruitful discussions.
[1] A. Khayari, A. T. Pérez and A. Castellanos, 2000 Conference on Electrical Insulation and Dielectric Phenomena, 470 (2002).

[2] C. Soria, A. Ramoz and A. T. Pérez, Europhys. Lett. 37, 541
(1997).

[3] M. Saint Jean, C. Even, and C. Guthmann, Europhys. Lett. 55(1), 45 (2001). 
[4] M. Saint Jean and C. Guthmann, J. Phys. : Condens. Matter 14, 13653 (2002).

[5] M. Saint Jean, C. Guthmann, and G. Coupier, Eur. Phys. J. B 39, 61 (2004).

[6] G. Coupier, C. Guthmann, Y. Noat and M. Saint Jean, Phys. Rev. E 71, 046105 (2005).

[7] P. Galatola, G. Coupier, M. Saint Jean, J.-B. Fournier and C. Guthmann, Eur. Phys. J. B 50, 549 (2006).

[8] G. Coupier, M. Saint Jean and C. Guthmann, Europhys. Lett 77, 60001 (2007).

[9] C. Coste, J.-B. Delfau, C. Even and M. Saint Jean, Phys. Rev. E 81, 051201 (2010).

[10] J.-B. Delfau, C. Coste, C. Even and M. Saint Jean, Phys. Rev. E 82, 031201 (2010).

[11] I. S. Aranson, D. Blair, V. A. Kalatsky, G. W. Crabtree, W.-K. Kwok, V. M. Vinokur and U. Welp, Phys. Rev. Lett 84, 3306 (2000).

[12] D. W. Howell, I. S. Aronson and G. W. Crabtree, Phys. Rev. E 63, 050301(R) (2001).

[13] I. S. Aranson, B. Meerson, P. V. Sasorov and V. M. Vinokur, Phys. Rev. Lett 88, 204301 (2002).

[14] M. V. Sapozhnikov, I. S. Aranson and J. S. Olafsen, Phys. Rev.
E 67, 010302(R) (2003).

[15] M. V. Sapozhnikov, A. Peleg, B. Meerson, I. S. Aranson and K. L. Kohlstedt, Phys. Rev. E 71, 011307 (2005).

[16] I. S. Aranson and J. S. Olafsen, Phys. Rev. E 66, 061302 (2002).

[17] M. V. Sapozhnikov, Y. V. Tolmachev, I. S. Aranson and W.K. Kwok, Phys. Rev. Lett. 90, 114301 (2003).

[18] I. S. Aranson and M. V. Sapozhnikov, Phys. Rev. Lett.92, 234301 (2004).

[19] M. V. Sapozhnikov, I. S. Aranson, W.-K. Kwok and Y. V. Tolmachev, Phys. Rev. Lett 93, 084502 (2004).

[20] K.-Q. Zhang and X. Y. Liu, J. Chem. Phys. 130, 184901 (2009)

[21] N. Vandewalle, G. Lumay, F. Ludewig, and J. E. Fiscina, submitted for publication (2011).

[22] J. C. Maxwell, A Treatise on electricity and magnetism (Dover Press, New York, 1891), Vol. I, pp. 268-276.

[23] A. T. Pérez, J. Electrostat. 56, 199 (2002).

[24] T. Gilet, N. Vandewalle, and S. Dorbolo , Phys. Rev. E79, 055201(R) (2009).

[25] N. Vandewalle, T. Gilet, and S. Dorbolo, in preparation (2011).

[26] M. Leconte, Y. Garrabos, F. Palencia, C. Lecoutre, P. Evesque, and D. Beysens, Appl. Phys. Lett. 89, 243518 (2006). 